\title{
Reliability and Latency in Binary Communication
}

\author{
Aslan Tchamkerten, Emre Telatar \\ LTHI-ISC-I\&C-EPFL \\ CH-1015 Lausanne, Switzerland \\ \{aslan.tchamkerten, emre.telatar\}@epfl.ch
}

\begin{abstract}
We consider the transmission of one of two messages over a Binary Symmetric Channels with perfect and instantaneous feedback. We study the situation where transmitter and receiver want to communicate reliably and quickly. We propose a simple decoding rule, and show that it minimizes the weighted combination of the probability of error and decoding delay for a certain range of crossover probabilities and combination weights.
\end{abstract}

\section{Statement of the Problem}

Suppose a customer wants to communicate to his stock broker either to buy or to sell a particular security. We assume that a penalty is associated to a misunderstanding, and at the same time the customer, say, in order to maximize his profit, wants to minimize the time it takes to send the message. The goals of being fast and being reliable are clearly contradictory, and we aim to investigate their tradeoff.

We formulate the simplest possible situation when the communication is carried out over a Binary Symmetric Channel with known crossover parameter $\varepsilon$ and perfect and instantaneous feedback. The transmitter chooses randomly one of two equally likely messages " 0 " and " 1 " and starts sending the all-zero sequence $x(0)=0,0, \ldots$ or the all-one sequence $x(1)=1,1, \ldots$ respectively, until the decoder makes a decision. We assume that there is decision feedback so that the transmitter knows when to stop.

At each instant $n$, the receiver computes the maximum likelihood probability of error $P_{e}^{\epsilon}\left(y_{1}^{n}\right)$ given the received symbols $y_{1}^{n}=y_{1}, \ldots, y_{n}$. We define the cost of decoding at time $n$ with the observation $y_{1}^{n}$ as

$$
\phi^{\alpha, \varepsilon, \rho}\left(y_{1}^{n}\right)=\alpha P_{e}^{\varepsilon}\left(y_{1}^{n}\right)^{\rho}+n,
$$

where $\alpha>0$ and $0<\rho \leq 1$ are fixed constants parametrizing the penalty should a message be wrongly decoded. Our aim is then to find a decoding rule minimizing the expected value of this cost function. More precisely let $\mathcal{S}$ be the set of all possible stopping times relative to the output sequence $Y_{1}, Y_{2}, \ldots$. We say that a stopping time $T^{*} \in \mathcal{S}$ is optimal if it satisfies

$$
\inf _{T \in S} \mathbb{E}\left(\phi^{\alpha, \varepsilon, \rho}\left(Y_{1}^{T}\right)\right)=\mathbb{E}\left(\phi^{\alpha, \varepsilon, \rho}\left(Y_{1}^{T^{*}}\right)\right)
$$

We shall consider only the case $0 \leq \varepsilon \leq \frac{1}{2}$. The situation $\frac{1}{2}<\varepsilon \leq 1$ is obtained by symmetry.

\footnotetext{
${ }^{1}$ The work presented in this paper was supported (in part) by the National Competence Center in Research on Mobile Information and Communication Systems (NCCR-MICS), a center supported by the Swiss National Science Foundation under grant number 5005-
} 67322 .

\section{A Short Sighted Optimal Strategy}

A stopping rule satisfying (1) can formally be computed via dynamic programming (see [2]). However this solution is difficult to characterize. The difficulty lies in the fact that, at each instant $n$, this stopping rule compares the current value of the objective function with values that may happen in the infinite future.

Consider now a stopping rule which compares the current value of the objective function to the expected value of the objective if it had stopped at the next step, and stops if the current value is better. This rule is in general suboptimal since it compares the current value only against a horizon that is one step ahead. The advantage of such a short sighted rule lies in its simplicity. Nonetheless, there are cases when it is best to be short sighted:

Theorem. For any $(\alpha, \varepsilon, \rho)$, let $T^{o}$ be the stopping time defined as

$$
T^{o}=\inf \left\{n \geq 1: \mathbb{E}\left(\phi^{\alpha, \varepsilon, \rho}\left(Y_{1}^{n+1}\right)-\phi^{\alpha, \varepsilon, \rho}\left(Y_{1}^{n}\right) \mid Y_{1}^{n}\right) \geq 0\right\}
$$

and let $u(\varepsilon)=\frac{1-\varepsilon}{\varepsilon}$.

i. For any $0<\rho<1$, and any $(\alpha, \varepsilon)$ such that

$$
\begin{aligned}
& \alpha \geq \frac{1}{\varepsilon^{\rho}-2^{1-\rho} \varepsilon(1-\varepsilon)-\left(\varepsilon^{2}+(1-\varepsilon)^{2}\right)^{1-\rho} \varepsilon^{2 \rho}} \quad \text { and } \\
& \alpha \leq \min \left\{2\left(1+u(\varepsilon)^{2}\right)^{\rho}, \frac{(1+u(\varepsilon))^{\rho} u(\varepsilon)^{\rho}}{u(\varepsilon)^{\rho}-1}\right\}
\end{aligned}
$$

$T^{\circ}$ is optimal.

ii. For any $0<\rho<1$, and any $(\alpha, \varepsilon)$ such that

$$
\begin{gathered}
\alpha<\frac{1}{\varepsilon^{\rho}-2^{1-\rho} \varepsilon(1-\varepsilon)-\left(\varepsilon^{2}+(1-\varepsilon)^{2}\right)^{1-\rho} \varepsilon^{2 \rho}} \quad \text { and } \\
0<\alpha \leq \min \left\{2(1+u(\varepsilon))^{\rho}, \frac{(1+u(\varepsilon))^{\rho} u(\varepsilon)^{\rho}}{u(\varepsilon)^{\rho}-1}\right\} \\
T^{o} \text { is optimal. }
\end{gathered}
$$

iii. For $\rho=1$ and any $(\alpha, \varepsilon)$ satisfying

$$
0<\alpha \leq \frac{1}{\frac{1}{2}-\varepsilon}
$$

$T^{o}$ is optimal.

\section{ACKNOWLEDGMENTS}

The authors thank Alon Orlitsky for many helpful discussions.

\section{REFERENCES}

[1] Robert G. Gallager, Information Theory and Reliable Communication, New York: John Wiley \& Sons, 1968.

[2] Y-S. Chow, H. Robbins and D. Siegmund, Great expectations: the theory of optimal stopping, Boston: Houghton Mifflin, 1971. 